\title{
Status Report on the detection of illicit materials in cargo containers by using neutron beams.
}

\author{
Giuseppe Viesti ${ }^{1}$ \\ Dipartimento di Fisica ed Astronomia dell' Università di Padova \\ Via Marzolo 8, 35131 Padova, Italy \\ E-mail: giuseppe.viesti@unipd.it
}

The search for explosives and dangerous or illicit materials hidden in cargo containers is one of the key tasks in countering illicit trafficking and terrorism. In this respect the use of neutron beams from portable accelerator has been studied in detail and some commercial systems are available. The state of the art of this technology is reviewed. Progresses in the last years are discussed making reference to the European programs EURITRACK and SMANDRA. The use of this technology in the search for SNM is also presented.

1

Speaker 


\section{Introduction}

Non-intrusive inspection of cargo containers has become a key issue in the fight against terrorism and illicit trafficking, since the shipping of cargo container between seaports represent today one of the more important way of the international trade. After 9/11, the Cargo Security Initiative [1] started setting standards for the cargo containers inspections in the trading between US and other countries. US are now aiming at $100 \%$ scanning of cargo containers shipped towards the American ports [2].

At present time, controls on cargo containers as well as on luggage in airports are mainly based on $\mathrm{X}$ - or gamma-ray scanners, which provide mainly the shape and density of the transported goods. Dual energy techniques have been also introduced to obtain a classification of the scanned object in some atomic number regions. An extensive review of the X-ray technology is presented in ref.3 in case of luggage inspection.

In seaport operations, additional technologies are welcome to clean/confirm X-ray alarms before the physical inspection of the transported goods. The opening of the cargo container is, indeed, a high cost, time consuming procedure. Non-destructive inspections with neutrons have been proposed in the past thanks to the neutron capability of penetrating materials and to produce reactions that provide qualitative and quantitative information on the elemental composition of the target. Moreover, the availability of different types of neutron source, including the electronic ones, was an important issue in the development of this technique.

From an historical point of view, one of the first use of neutrons to detect explosives in airports was based on the so called Thermal Neutron Analysis (TNA) [4], looking to the high energy capture gamma rays from nitrogen nuclei as a signature of the presence of explosives. The limit of the technique is the high nitrogen content in some benign materials thus generating false alarms. On the other side, the Fast Neutron Analysis (FNA) identifies the elements though the gamma rays produced mainly in inelastic scattering reactions induced by $14 \mathrm{MeV}$ neutrons produced by electronic generators using the $\mathrm{D}+\mathrm{T}$ fusion reaction. Systems were proposed combining FNA with TNA to maximize the number of detectable elements [5]. Finally, the use of $14 \mathrm{MeV}$ tagged neutrons, i.e. using the associated particle technique, was providing an improved signal-to-noise ratio with the possibility of extracting elemental information from a well defined voxel inside the target [6]. The aim of this paper is to present a status report in the use of neutrons as an additional tool in the inspection of cargo container, discussing the recent progress and the problems related to this technology. 


\section{The state of the art}

Several projects devoted to the detection of illicit materials inside cargo containers or trucks have been completed during the last decade [7]. Some prototypes passed successfully the laboratory characterization and went to intensive field-testing. It is quite difficult to provide a complete list of projects because some of them were performed with commercial or security restrictions and final results were not publically presented. However some qualitative tracking of the different projects is available by looking to conference proceedings in the field of application of accelerators.

It is worth mentioning that a large system based on the so called PFNA (Pulsed Fast Neutron Analysis) [8] to be used as first line scanning systems of large targets (trucks) was not passing the field tests.

During 2007-8, the FP6 EU funded project EURITRACK [9] was completed with the fielding of a prototype portal for second line inspection. This portal makes use of the so-called Tagged Neutron Inspection System (TNIS). The EURITRACK basic concept was to inspect with neutrons only selected voxels inside cargo containers identified in the first-line X-ray scan. The EURITRACK portal was installed and operated at the Rijeka seaport for more than two years, producing a database of about 150 inspected objects $[10,11]$. The EURITRACK portal at the Rijeka seaport is shown in Fig.1.

Today few neutron-based system are commercially available. Two of them make use of the tagged neutron technique: ULIS, a small parcel inspection system developed by SODERN [12], and SENNA, developed by the APSTEC Company [13]. Moreover, a commercial line of products of the ECIL-RAPISCAN Company makes use of the TNA technique [14].

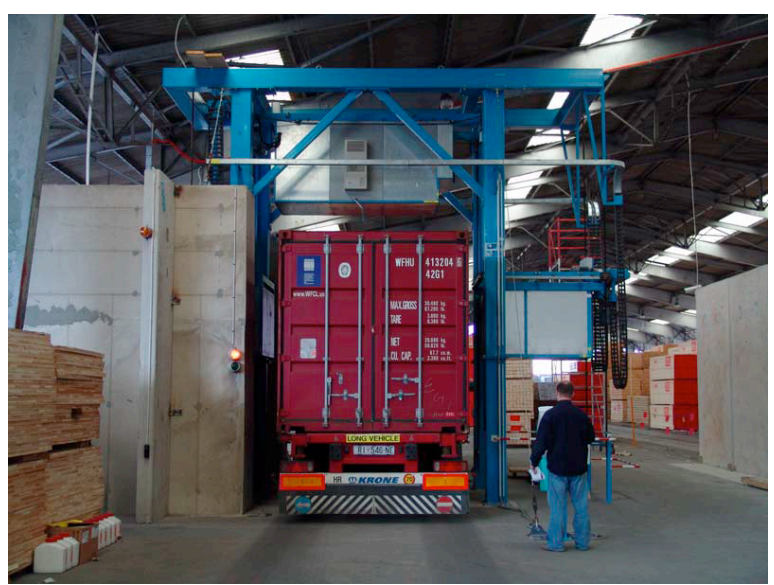

Fig.1 The EURITRACK portal

More recently a small prototype of Tagged Neutron Inspection system was developed and tested within a national Italian project to study the possibility of a second generation system with extended capabilities from the search for illicit and explosive material to the detection of special nuclear material (SNM). This prototype, called 
SMANDRA was used only in laboratory experiment [15]. A picture of the SMANDRA system is shown in Fig.2.

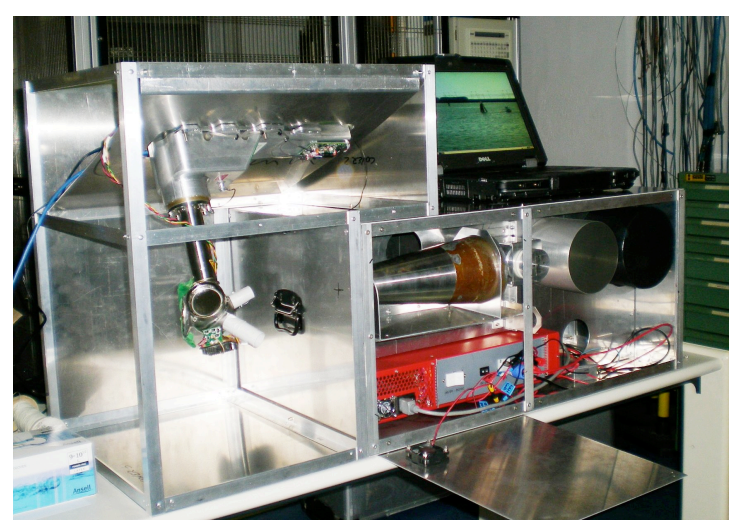

Fig.2 The SMANDRA system without external case. The left box contains the neutron generator whereas shadow bar, detectors and electronics are in the right box. On top of the right box is located the on-board PC.

In the following, the strengths and weaknesses of the neutron-based technologies are discussed making special reference to EURITRACK and SMANDRA projects.

\section{General aspects of the neutron techniques}

The strength of the TNIS technology is essentially due to the capability of obtaining signatures from the nuclear species inside a well-defined voxel inside cargo containers also in case of sealed systems as tankers. The non-destructive inspection of a sealed system is obviously not possible with other competing technologies in the detection of explosives as those based on vapor sensing. The best demonstration of this capability was provided in the work of Pesente [6] in which it was documented the possibility of separating the signal from the explosive inside an anti-tank landmine from the external case. This strength need, however, to be evaluated taking into account two constrains: the counting statistics and the specificity of the derived information.

The TNIS offers the possibility of varying the size of the inspected voxel by two technical features. The size of the neutron beam (at a given depth) can be tailored by using a pixelized associated alpha particle detector. This electronic focusing was obtained in EURITRACK by using a matrix of $8 \times 8$ scintillators [16]. The average position of the inspection voxel inside the container and its width along the average neutron path is selected by setting neutron time-of-flight window. Precision in this task is associated with the time resolution of the system. However, the technical possibility of minimizing the voxel implies the reduction of the number of target nuclei. Consequently a sufficient counting statistics in the gamma-ray spectra is obtained by using a higher neutron flux or a longer measure time. In the real world application, the 
maximum neutron flux is limited by licensing procedure and the typical inspection time is determined by the standard seaport operation.

The licensing problem is an important issue. Within the current EU legislation it is highly unrealistic to license a neutron-based system when the $4 \pi$ neutron flux exceeds the $10^{7}$ neutron/s limit. For larger neutron flux the complexity of the procedure, the need of heavy biologic shield and large exclusion area make very hard to operate such system in seaports. Typical operating conditions of the EURITRACK prototype were indeed a neutron flux of $10^{7}$ neutron/s and a measure time of $600 \mathrm{~s}$. It is worth noting that in principle higher neutron can be used without creating problems in the irradiation of foods and medical stuff [17]. As a result, the possibility of tuning the neutron beam size was not used in field operations whereas the time windowing is essential to slice in depth the target.

The second point is the specificity of the information obtained from the nuclearbased techniques. The large majority of the proposed systems extract the elemental composition from the gamma ray spectra resulting from the irradiation. The information consists in the relative yield of light elements, usually carbon, oxygen and nitrogen in case of explosive search. Some systems are also able to determine the hydrogen content by looking to the neutron capture reaction on hydrogen. The extracted elemental ratios are then compared, in a simple approach, with a reference table that allows checking the type of material and thus the agreement with the shipping manifest.

In case of large object as cargo container it is worth considering some additional problems. Generally the goods might have different type of packaging that is not clearly described in the shipping manifest and might influence the identification of the material itself. In a large size target, neutrons are slowed down by travelling inside the material. The yield of specific gamma rays is normally a function of the neutron energy. Consequently the yield of the gamma rays might change with the depth inside the cargo with the need of material- and depth-dependent corrections. Moreover, some gamma rays are attenuated in travelling from the production point to the detector [18]. This means that, in the cargo container inspection, specific software corrections are playing an important role to obtain a realistic material identification. 


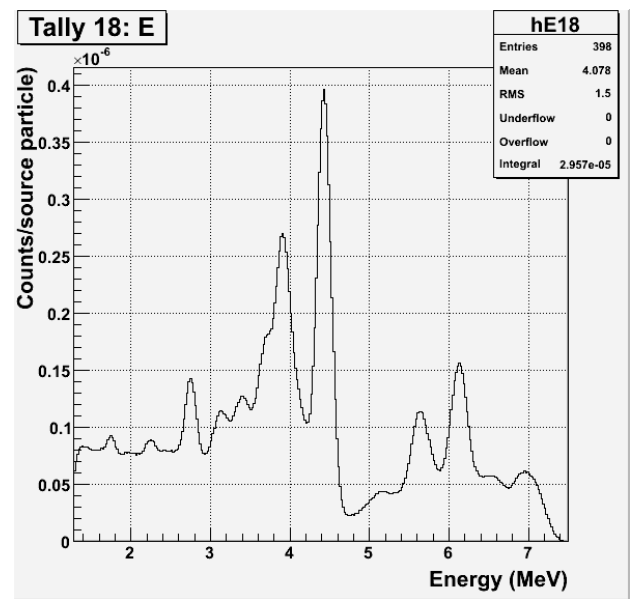

Fig.3 Monte Carlo simulated spectrum from TATP irradiation.

A second important point is that the elemental composition of some explosives is very close to commonly used materials and the presence of illicit materials is identified clearly only when the illicit and the benign material have different composition. As an illustration of the problem, the case of the homemade explosive TATP is considered. The Monte Carlo simulated spectrum from a TATP sample is presented in Fig.3, showing the characteristic gamma rays from ${ }^{12} \mathrm{C}(\mathrm{E} \gamma=4.4 \mathrm{MeV})$ and ${ }^{16} \mathrm{O}(\mathrm{E} \gamma=6.1 \mathrm{MeV})$. The chemical formula of TATP is $\mathrm{C}_{6} \mathrm{H}_{12} \mathrm{O}_{4}$ with a $\mathrm{C} / \mathrm{O}$ ratio very close to a variety of common materials, including cotton. Consequently, it is rather difficult to discriminate clearly TATP using neutron probes which determine only the $\mathrm{C} / \mathrm{O}$ ratio, when the presence of other common use material can't be a priory excluded [19].

\section{Technical developments}

The EURITRACK prototype design was realized in 2005 using top technologies available at that time. Since then technological developments in the field of neutron generators, detectors, front end-electronics and computing have been quite impressive. The EURITRACK SODERN neutron generator was developed starting from the GENIE product line with the inclusion of the associated particle detector. The neutron emitting tube has a length of about $60 \mathrm{~cm}$ and weight of about $30 \mathrm{~kg}$. The neutronemitting unit was connected by $15 \mathrm{~m}$ long cables (including the high voltage cable) with a cabinet containing control modules and high voltage generator. This cabinet has a weight of about $140 \mathrm{~kg}$. In SMANDRA the new miniaturized neutron generator TPA-17 developed by SODERN for the ULIS system was used, shown in the left box of Fig.2. This is an extremely compact generator with on-board control cards and high voltage 
generator. The new neutron generator is lightweight and low power so that it can be used in transportable, battery operated inspection system.

The second field of strong technical development is the front-end electronics. The EURITRACK front-end [20] was realized using high-density VME CAEN cards [21]. The read-out was designed by using typical analog circuitry: CFTD and TDC for time measurements, QDC with integration gates for energy measurements. The trigger of the system was built starting from a dedicated card. A separate High Voltage Power Supply system was used to bias the detector PMTs. The acquired data is then processed off-line by dedicated software. In the more recent SMANDRA system, the detector signals are sent directly to VME CAEN fast digitizers and also HV power supply in VME standards is used $[15,22]$. The fast digitizer contains a FPGA for on-line processing of the registered signal, producing directly a time stamp that can be used for filtering coincidence events, the energy integration of the pulse and a partial integration that is used for pulse shape discrimination analysis with liquid scintillators. Moreover a selected portion of the digitized signal can be stored for precision time measurements in the off-line analysis by using Virtual CFTD. Intensive laboratory measurements have demonstrated that the energy resolution achievable by digital signal processing is better than the one characteristic of analog circuitry. On the other hand the time resolution achieved with virtual CFTD is lightly worse with respect to the one characteristic of classical CFTD modules [22]. However time resolutions lower than 5 ns have been obtained, value that is well within the need of cargo container inspections. Consequently the full front-end can be build using a small number of digitizer and HV power supply VME cards, replacing the EURIRACK analog electronic front-end. The reduction of the volume and weight of the electronics, together with the lowering of the power consumption make possible to mount directly the front-end on board the inspection system and operate it with batteries. The VME mini-crate that hosts the front-end and HV cards is visible in the right box of Fig. 2.

The use of digital electronics requires a suitable on-board computing system to initialize the system, operate the data acquisition and then perform the data analysis. In the SMANDRA system this is obtained by a LINUX commercial ruggedized portable computer that perform all functions. The control of the neutron generator might be implemented also on this computer. Wireless communication tools allow connecting the on-board computer with a second identical remote unit that is used to operate the system outside the exclusion area. Further developments make use of web-based technologies to connect the on-board computer with any type of operator device, including smartphones.

Finally, EURITRACK used 22 large $\mathrm{NaI}(\mathrm{Tl})$ scintillators distributed in three arrays: the REFLECTION array on the same side of the neutron generator, the TRANSMISSION array on the opposite side and the TOP array containing 18 detectors 
placed right above the container. The $\mathrm{NaI}(\mathrm{Tl})$ detector was selected as a good compromise between intrinsic efficiency and cost. It is worth mentioning that the field operations suggest the following points: a) due to the attenuation of the neutron beams useful information is obtained only for the first half of the container thickness; $b$ ) due to the latter effect, the TRANSMISSION array and part of the TOP detectors are too far from the interaction point to contribute significantly to the total counting statistics. Consequently the detectors that are providing sizeable contribution are the ones mounted in reflection geometry. This fact motivates the geometrical design of the SMANDRA system with all detectors mounted in reflection geometry.

In other recent systems, including SMANDRA, a new type of scintillator, the $\mathrm{LaBr}(\mathrm{Ce})$ crystal, was used. This crystal is characterized by a very high energy resolution (about $3.5 \%$ at $661 \mathrm{keV}$ to be compared with $6 \%$ characterizing $\mathrm{NaI}(\mathrm{Tl})$ ) and sub-nanosecond time-resolution [22]. Such detectors are characterized by limited volume and very high price. However, it is very convenient when the non-destructive inspection system is operated both in active mode (i.e. using the neutron generator) and in passive mode as an high efficiency spectrometric system. This is an essential point for future inspection system that needs to include the search of special nuclear material in their capabilities.

SMANDRA was designed for this dual use search and includes, together with large volume $\mathrm{NaI}(\mathrm{Tl})$ and high resolution $\mathrm{LaBr}(\mathrm{Ce})$ also detectors for fast neutrons (liquid scintillator) and thermal neutrons (3He proportional counter). As reported in ref. 23, the search of special nuclear material benefits from the possibility of detecting in passive mode neutrons and gamma rays. In the case of search for SNM with low intrinsic neutron emission, as U samples, passive measurements are very difficult and the use of tagged neutron inspection is needed. However, in case of heavy targets, the neutron induced gamma-ray spectra do not show signatures that allow identifying the elements in the sample. It has been verified that, in this case, correlations between the coincident neutrons and gamma rays stimulated by the fast neutron irradiation are a good tool to distinguish $\mathrm{SNM}$ from other heavy samples, as $\mathrm{Pb}$. The discrimination between uranium and lead is particularly interesting since those objects might provide a very close signature in a X-ray image.

\section{Conclusions}

The neutron-induced inspection techniques have been developed in the past essentially to search for explosive and illicit material in cargo containers. Results from past experience demonstrate that such systems can be hardly used as first-level inspection tool but are very useful as second line inspection.

Results form the EURITRACK project, in which only selected voxels inside risky cargos were inspected, demonstrated the capability of the neutron technology. The 
design of the EURITRACK system, i.e. a fixed portal with large exclusion area and heavy biological shielding, was implying high development and maintenance costs. It was one of the major limiting factors in the lack of commercial follow up of the project.

Due to the realistic limits in term of neutron flux and measurement time of the neutron technology, it seems that second generation tagged neutron inspection systems might be designed to reach the following goals:

- use of mobile systems mounted on remote-controlled special vehicles (as forklift) to position the neutron head and inspect specific voxels in cargo after the X-ray scan;

- use of restricted access area controlled by interlocks to perform the neutron irradiation but without need of fixed biological shielding;

- use of high-density digital electronics and miniaturized neutron generators operated by an on-board computer with wireless connection with the operator remote device placed outside the exclusion area;

- use of high-resolution gamma ray and fast neutron detectors to operate the system also as passive radiation spectrometer.

In this way the same system, as in the case of SMANDRA, can be used for explosive and illicit material as well as for SNM search, increasing the added value of this technology. Once again, non-destructive inspection by using neutrons represents an useful tool to inspect also sealed objects that are hardly analyzed with other technologies.

\section{References}

[1] see http://www.cbp.gov/xp/cgov/trade/cargo_security/csi/ and documents therein

[2] see http://www.hlswatch.com/2012/07/16/scanning-cargo-containers-a-stickyissue/

[3] K. Wells, D.A. Bradley, Applied Radiation and Isotopes 70 (2012) 1729

[4] see as example P. Shea, T. Gozani, H. Bozorgmanesh, Nucl. Instr. Meth. A299 (1990) 444

[5] G. Vourvopoulos, F.J. Schultz, Nucl. Instr. Meth. B79 (1993) 585

[6] S. Pesente et al., Nucl. Instr. Meth. A531 (2004) 657 and referenced therein.

[7] See as example T. Gozani, Nucl. Instr. Meth. B213 (2004) 460 and references therein.

[8] J. Rynes et al., Nucl. Instr. Meth. A422 (1999) 895

[9] B. Perot et al. Nucl. Instr. Meth. B 261 (2007) 295

[10] C. Carasco et al, Nucl. Instr. Meth. A588 (2008) 397

[11] J. Obhodas et al, Nucl. Instr. Meth. B A619 (2010) 460

[12] See http://www.sodern.com/sites/en/ref/ULIS_80.html 
[13] See http://www.apstec.ru/pr31.phPp?kuna=en

[14] See http://www.ecilrapiscan.com/static/mob_veds.htm

[15] D. Cester et al., AIP Conf. Proc. 1423, pp. 335-340; doi:http://dx.doi.org/10.1063/1.3688822

[16] S. Pesente et al., Nucl. Instr. Meth. B261 (2007) 268

[17] E. Giroletti et al., Radiation Physics and Chemistry, 81 (2012) 758

[18] C. Carasco et al., Nucl. Instr. Meth. A582 (2007) 638

[19] G. Viesti et al., Search of Explosives in Vehicles by Using Tagged Neutrons, in Detection of Liquid Explosives and Flammable Agents in Connection with Terrorism, H. Schubert and A. Kuznetsov editors, 2008 Springer, The Netherlands.

[20] M. Lunardon et al., Nucl. Instr. Meth. B261 (2007) 391

[21] See http://www.caen.it/

[22] L. Stevanato et al., Nucl. Instr. Meth. A678 (2012) 83

[23] D. Cester et al., Nucl. Instr. Meth. A663 (2012) 55 